\title{
ON THE CONFORMATIONAL POTENTIALS OF
}

\section{BIOMOLECULES}

\author{
Shujun Wang \\ Brisbane, Australia, QLD 4074 \\ shujunwangresearch@gmail.com
}

\section{Abstract}

Biological molecules such as proteins, DNA and RNA have specific spatial configuration known as conformation which is essential for the functions of those molecules. The specific binding or 'docking' of one molecule (e. g. $\mathrm{O}_{2}$, glucose, proteins) to its conformational matching biomolecules (e. g. DNA, RNA, proteins) underscores all biological processes ranging from DNA transcription, protein synthesis, biological signaling (e. g. endocrinology), in vitro transportation of nutrition to enzymatic reactions and so on. However, the mathematical or physical basis of such 'shape-driven' binding behaviors still remains mystery. In particular given the stochasticity and the crowdedness of the intracellular environment, how cell manage to achieve all the molecule binding in biological processes is not yet well understood. Here, we present a theory that may provide a footing for mathematical or physical understanding of the binding behavior of molecules with biomolecules. This theory attributes the binding interactions to the existence of conformational potentials that are specific to the shapes of the molecules. We derived a spatially varied diffusion coefficients and the corresponding stochastic equation for diffusive motion of molecules in a conformational potential, which indicates the Einstein's diffusion theory featured with an invariant diffusion coefficient is an approximation of our version of diffusion at places far from the centers of the binding sites. The theory provides a good explanation for the 'anomalous diffusion' behavior of biomolecules observed in single particle tracking studies. It also provides for the first time an experimentally measurable physical quantity named 'conformational coefficient' for studying the conformational behaviors and functioning mechanisms of biomolecules.

\section{Conformational potential}

Intracellular biological processes such as DNA transcription and translation, cell metabolism, molecular transports require some molecules (e. g. small common molecules or biomolecules) to bind specifically to certain biomolecules (e. g. DNA, RNA, proteins). It is known that such binding or 'docking' behavior is dominated by the conformation (i. e. shape) of the biomolecules $^{1-2}$. The binding behavior is normally called 'induced fit' or 'conformational selection' ${ }^{3-5}$. The code of conduct in modern drug discovery is to search for biocompatible molecules with certain desired 'shapes' that match a critical protein in a biological process ${ }^{6}$. The binding of the drugs to a targeted biomolecule prevents the binding of a necessary component to it (e.g. proteins) breaking up a biological process (e. g. the biological signaling process for rising body temperature) underpins the function of drugs. It is well know that the intracellular environment is over crowded with biomolecules (up to $40 \%$ of all intracellular mass) ${ }^{7-9}$. The shape driven binding behavior is very specific and is essential for all the sophisticated reactions taken place in a cell. Many of the substances vital to life depend on diffusion or 'random walk' to get to the 'binding sites' of biomolecules. Putting all these pieces 
of information together, a central question is how cell manage to achieve all the molecular binding within such a crowd environment. With the type of precision, mechanistic cleverness, complexity and efficiency we observed in biological studies, it is difficult to believe all these binding events are just the work of 'random walk'. We believe the answer may rely on the existence of a shape driven potential which are able to guide the molecules toward the binding sites under the background random forces.

When two objects automatically interact with each other at a distance (i.e. finding a binding site and bind), intuitively, one may consider there is some form of physical 'field' existing in between the two objects and the interactions (e. g. forces) is a product of the 'field', like gravitational field between an apple and the Earth, magnetic field in between two magnets, electrostatic fields in between charged particles. As origin of all well known theories, we also begin our theory from such a simple intuition that there must be shape-driven potential fields exist in between the interacting biomolecules originating from the centres of the binding sites. We name it the 'conformational potential'. Like all potential, this potential should satisfy the Poisson's equation, one can apply Gauss's theorem, eventually, arrives at a formula showing the potential is inversely proportional to the distance from the binding centre of a 'host' biomolecule. To simplify the development of our theory, let us define a physical quantity called 'conformational coefficient' $\xi$ for which the conformational potential $\varphi_{\mathrm{c}}$ takes the form,

$$
\varphi_{c}=\frac{\xi}{r}
$$

The corresponding force $\mathbf{F}(r)$ a conformational matching molecule at position $\mathbf{r}$ feels is therefore,

$$
\mathbf{F}(r)=-\nabla \varphi_{c}
$$

Molecules inside cells swim in a liquid environment. At the absence of an external force, a molecule undergoes diffusion governed by free Brownian motion a stochastic process that can be well described by Albert Einstein's diffusion theory. However, we argue that, if there is a force in between two conformational matching molecules, the diffusion process must be different from the free form. Namely, one should be able to examine the conformational potential via studying the diffusion behavior of molecules in cells. Following we will develop a diffusion theory based on the postulation of the existence of conformational potentials.

\section{Fork-Planck equation for stochastic diffusion} The most widely adopted theory for studying intracellular diffusion of biomolecules is Einstein's diffusion theory ${ }^{10}$. Einstein was among the first to apply the Fokker-Planck equation to study Brownian motion in which diffusion played a critical role. We also started our formulation of the diffusion of biomolecules in the presence of conformational potential on a Fokker-Planck equation ${ }^{11-12}$.

$$
\frac{\partial p}{\partial t}=\nabla \cdot\left(\nabla D p-\frac{\mathbf{F}(r)}{\gamma} p\right)
$$

Where $p$ is the probability density function of the position of the molecule, $\mathrm{D}$ is the diffusion coefficient, $\gamma$ is the amplitude of the friction force.

For an arbitrary volume $\Omega$, the number of molecules $\left(\mathrm{N}_{\Omega}\right)$ at time $\mathrm{t}$ within this volume can be expressed as the following integral,

$$
\frac{\partial N_{\Omega}}{\partial t}=\int_{\Omega} d r \nabla \cdot\left(\nabla D p-\frac{\mathbf{F}(r)}{\gamma} p\right)
$$

Applying Gauss law, the volume integral is replaced by a surface integral over $\partial \Omega$,

$$
\frac{\partial N_{\Omega}}{\partial t}=\int_{\partial \Omega} d \mathbf{s} \cdot\left(\nabla D p-\frac{\mathbf{F}(r)}{\gamma} p\right)
$$

Therefore, we can extract the flux through the 
surface $\partial \Omega$ as,

$$
\mathbf{j}=\left(\nabla D p-\frac{\mathbf{F}(r)}{\gamma} p\right)
$$

As pointed out by Einstein, the flux vanishes at thermal equilibrium,

$$
\nabla D p-\frac{\mathbf{F}(r)}{\gamma} p=0
$$

Besides, at thermal equilibrium, the probability distribution function can be described by the Boltzmann distribution,

$$
p=\frac{1}{Z} e^{-\beta \varphi}
$$

Where $\mathrm{Z}$ is the partition function, a normalization factor and $\beta=1 / \mathrm{K}_{\mathrm{B}} \mathrm{T} . \quad \mathrm{K}_{\mathrm{B}}$ is the Boltzmann constant. Therefore, one arrives at,

$$
e^{-\beta \varphi}\left(D \beta \mathbf{F}(r)+\nabla D-\frac{\mathbf{F}(r)}{\gamma}\right)=0
$$

Which indicates the following equation holds at thermal equilibrium,

$$
D \beta \mathbf{F}(r)+\nabla D-\frac{\mathbf{F}(r)}{\gamma}=0
$$

Rearrange this equation, one obtains

$$
\nabla D=\left(\frac{1}{\gamma}-D \beta\right) \mathbf{F}(r)
$$

\section{Diffusion coefficient at the presence of a conformational potential}

The main feature of Einstein's diffusion theory is that the diffusion coefficient D is spatially invariant. This treatment is generally valid for 'free' diffusion where particles are not subject to external forces or those forces are negligible. Einstein's diffusion theory is most popularly applied in studies of intracellular diffusion of biomolecules at the moment but often meets significant disagreement with experimental data which will be discussed in later section.

Here we argue that when the molecules are in the vicinity of a spatially varied potential field (e. g. a conformational potential), assumption of spatially invariant diffusion coefficients might not be valid. At such circumstances, we argue that the diffusive motion will be significantly dominated by the potential field. Indeed, given that $\mathbf{F}(r)=-\nabla \varphi_{\mathrm{c}}$, Eq. (1) supports our argument. Moreover, in the vicinity of the conformational potential field, the amplitude $\gamma$ of friction force will be very large as it is related to $\mathbf{F}(r)$ by the Langevin equation ${ }^{11-12}$, namely $1 / \gamma$ will be very small. Therefore, Eq. (1) can be well approximated by the following equation which is a partial differential equation with respect to D.

$$
\nabla D=-\beta D \mathbf{F}(r)=\beta D \nabla \varphi_{c}
$$

Although we arrived at Eq. (2) through an approximation, later we will see Eq. (2) is essential for describing diffusion in the presence of a conformational potential as solving Eq. (2) will not just provide us a spatially dependant diffusion coefficient but also reveals that at places far from the centre of conformational potentials, the diffusion can be well approximated by the Einstein view of diffusion. Following we will provide the solutions to this equation in $1 \mathrm{D}, 2 \mathrm{D}$ and $3 \mathrm{D}$.

\section{$1 D$ solution}

The 1D Eq. (2) takes the following form,

$$
\frac{\partial D}{\partial x}=-\frac{\beta \xi}{x^{2}} D
$$

It can be solved analytically,

$$
\int \frac{1}{D} d D=\int-\beta \xi x^{-2} d x
$$

The solution is

$$
D=e^{\frac{\beta \xi}{|x|}+C}
$$

From this solution, it is immediately obvious that the diffusion coefficient $\mathrm{D}$ becomes a constant at places far from the centre of the 
conformational potential, which implies that the spatially invariant Einstein's diffusion coefficient is an approximation of the diffusion coefficient derived here at the 'far end' from the centre of the conformational potentials. Or in other words, the Eq. (2) has the boundary condition,

$$
\begin{aligned}
& x \rightarrow \infty \\
& D=D_{\infty}
\end{aligned}
$$

Where $\mathrm{D}_{\infty}=\mathrm{e}^{\mathrm{C}}$ is the spatial invariant free diffusion coefficient described by Einstein's diffusion theory. Hence, we can rewrite the 1D solution as,

\section{$2 D$ solution}

$$
D=D_{\infty} e^{\frac{\beta \xi}{|x|}}
$$

The 2D Eq. (2) takes the form,

$$
\begin{aligned}
& \frac{\partial D}{\partial x}=-\beta \xi \frac{x}{\left(x^{2}+y^{2}\right)^{3 / 2}} D \\
& \frac{\partial D}{\partial y}=-\beta \xi \frac{y}{\left(x^{2}+y^{2}\right)^{3 / 2}} D
\end{aligned}
$$

The solution is,

$$
D=D_{\infty} e^{\beta \xi \frac{1}{\sqrt{x^{2}+y^{2}}}}
$$

\section{$3 D$ solution}

The 3D Eq. (2) has the form,

$$
\begin{aligned}
& \frac{\partial D}{\partial x}=-\beta \xi \frac{x}{\left(x^{2}+y^{2}+z^{2}\right)^{3 / 2}} D \\
& \frac{\partial D}{\partial y}=-\beta \xi \frac{y}{\left(x^{2}+y^{2}+z^{2}\right)^{3 / 2}} D \\
& \frac{\partial D}{\partial z}=-\beta \xi \frac{z}{\left(x^{2}+y^{2}+z^{2}\right)^{3 / 2}} D
\end{aligned}
$$

The solution is,

$$
D=D_{\infty} e^{\beta \xi \frac{1}{\sqrt{x^{2}+y^{2}+z^{2}}}}
$$

Therefore, the generalized solution for $n$ dimension is,

$$
D=D_{\infty} e^{\beta \xi \frac{1}{r}}
$$

Where $r$ is the distance from the centre of the binding site to the position of the molecule.

Diffusive motion of a molecule in the presence of a conformational potential

As mentioned before, Einstein's diffusion theory offers a beautiful description for the free form of diffusion where the diffusion coefficient $\mathrm{D}$ is spatially invariant which is generally applicable in many cases (i. e. sugar molecules in water). The corresponding diffusion process is governed by a stochastic differential equation derived from Fokker-Planck equation forming the Einstein's view of Brownian motion ${ }^{10}$.

$$
\frac{\partial p}{\partial t}=D \nabla^{2} p
$$

A less well known diffusion process is the Smoluchowski Diffusion ${ }^{13}$ which describes diffusive motion taken place in a potential field. Similar to Einstein's diffusion, it also has its origin from Fokker-Planck equation but offers a different view upon the Brownian motion governed by a different stochastic differential equation.

$$
\frac{\partial p}{\partial t}=\nabla \cdot D(\nabla-\beta \mathbf{F}(r)) p
$$

Nevertheless, it is often considered the two versions of diffusion or Brownian motions are equivalent especially when spatially invariant diffusion coefficient is assumed ${ }^{12,14}$. However, Smoluchowski's view is broader as the equation does indicate D can be spatially varied. Here, we take the view of Smoluchowski and apply what we have achieved on the formulation of diffusion coefficient in the presence of a conformational potential to derive our version of stochastic equation for the diffusive motion of a molecule in the vicinity of a biomolecule. We obtain the following expression.

$$
\frac{\partial p}{\partial t}=D\left(\nabla^{2} p+\mathbf{a} \cdot \nabla p+b p\right)
$$


Where,

$$
\begin{aligned}
& D=D_{\infty} e^{\frac{\beta \xi}{r}} \\
& \mathbf{a}=2 \beta \nabla \varphi_{c} \\
& b=\beta \nabla^{2} \varphi_{c}+\beta^{2} \nabla \varphi_{c} \cdot \nabla \varphi_{c}
\end{aligned}
$$

The 1D form of Eq. (4) is,

$$
\frac{\partial p}{\partial t}=D_{\infty} e^{\frac{\beta \xi}{x}}\left(\frac{\partial^{2} p}{\partial x^{2}}-\frac{2 \beta \xi}{x^{2}} \frac{\partial p}{\partial x}+\left(\frac{2 \beta \xi}{x^{3}}+\frac{\beta^{2} \xi^{2}}{x^{4}}\right) p\right)
$$

From this equation, it is apparent that at the 'far end' $(r \rightarrow \infty)$ the diffusive motion of the molecule can be well approximated by the Einstein's view of Brownian motion. However, Einstein's view is no longer valid when the molecule is close to the centres of the conformational potential of biomolecules.

\section{Experimental determination conformational coefficient $\boldsymbol{\xi}$}

The as derived spatially varied diffusion coefficient (Eq. (3)) provides a passage for experimentally determination of the value of the conformational coefficient $\xi$ of biomolecules (e. g. proteins) via measurement of the diffusion coefficients. Rearranging Eq. (3), we can have either,

$$
\xi=\frac{r}{\beta} \ln \left(\frac{D(r)}{D_{\infty}}\right)
$$

or,

$$
\ln \left(\frac{D(r)}{D_{\infty}}\right)=k r^{-1} \quad \text { where, } \quad \xi=\frac{k}{\beta}
$$

Therefore, one can design experiments to measure the 'global' diffusion coefficients $\mathrm{D}_{\infty}$ and the diffusion coefficients $\mathrm{D}(\mathrm{r})$ of a conformational matching molecule in the vicinity of a biomolecule in a viscous aqueous media to determine $\xi$ (Eq. (5)) or, $\xi$ can be extracted by linear fitting the curve in the $\ln \left(\mathrm{D}(\mathrm{r}) / \mathrm{D}_{\infty}\right)$ and $\mathrm{r}^{-1}$ plane (Eq. (6)). An equivalent measurement is that one can measure in the same media the diffusion coefficients of molecules with $(\mathrm{D}(\mathrm{r}))$ and without $\left(\mathrm{D}_{\infty}\right)$ the presence of their conformational matching biomolecues to determine $\xi$. Given the advances in the single particle tracking and the resolution of such experiment has been improved to $20 \mathrm{~nm}^{15-19}$, such an experiment to directly test the existence of the conformational potential and measure the magnitudes of the $\xi$ of biomolecules should not be difficult to conduct.

\section{Discussion}

\section{Conformational potentials and intracellular anomalous diffusion}

We started on the intuition that the binding of two conformational matching molecules are dominated by a specific conformational potential between the two molecules. This might create the impression that the force arises in between two biomolecules from the conformational potential is an attractive force. However, there is no explicit indication in our theory so far that the force experienced by a molecule in the conformational potential of a biomolecule is only attraction. Indeed from Eq. (5), the conformational coefficient $\xi$ can take either positive or negative values depending on the ratio of $\mathrm{D} / \mathrm{D}_{\infty}$. Therefore, we argue that the conformational potential may be ubiquitous among biomolecules and only conformationally matching molecules experiencing 'attractive forces', otherwise, repulsion exist in between nonmatching molecules. This can explain the specificity of biomolecules in binding events, namely, each type of biomolecules only binds to molecules with a specific shape. Another point is the ubiquitous repulsion due to conformational potential among nonmatching biomolecules can probably also afford an explanation for the reason why all the biomolecules can function so well in an over-crowded intracellular environment conducting sophisticated biological processes and reactions without agglomeration, sedimentation as observed often in artificial 
nano- and microscale materials.

Besides, the existence of 'attractive' and 'repulsive' forces among biomolecules as a result of conformational potentials may also affords an explanation for the 'anomalous diffusion' observed in intracellular single particle tracking studies ${ }^{20-23}$. In general, it is widely known that the intracellular diffusion of biomolecules deviates significantly from Einstein's free Brownian motion theory which is named 'anomalous diffusion'. In an anomalous diffusion, it is often noticed that biomolecules either undergo so called 'super-diffusion' where the measured diffusion coefficients are greater than the Einstein's Brownian motion value, or 'under diffusion' where the measured diffusion coefficients are lower than Einstein's Brownian motion value. Moreover, empirical relations have been identified showing the diffusion coefficients having exponential relationship with tracking time $^{23-25}$. Currently, such divergences have been attributed to postulations including 'friction', 'spatial confinement within cells' etc ${ }^{25-28}$.

The anomalous diffusion may be explained with the spatially varied diffusion coefficient (Eq. (3)) derived in this paper. Briefly, we can consider the exponential part of the Eq. (3) as a weight factor, a molecule can either have super-diffusion in case of the exponential factor greater than 1 (i. e. matching conformation) or experience sub-diffusion when the exponential factor is smaller than 1 (i. e. nonmatching conformation) in the vicinity of a conformational potential. When the molecules are not in the vicinity of conformational fields, they approach the free Brownian diffusion. Nevertheless, such a simple qualitative analysis has to be justified in the context of intracellular environment which is full of biomolecules. Following, we provide a formulation to describe the $3 \mathrm{D}$ intracellular diffusion in the presence of $\mathrm{n}$ biomolecules based on what we developed so far.
Let us assume, at time $t$, there are $n$ biomolecules at positions $\left(a_{1}, b_{1}, c_{1}\right),\left(a_{2}, b_{2}\right.$, $\left.c_{2},\right), \ldots,\left(a_{n}, b_{n}, c_{n}\right)$ with their conformational coefficients $\xi_{1}, \xi_{2}, \ldots, \xi_{n}$. Following what has been established in previous section, one should be able to get the full description of diffusion coefficient as below for a molecule at position $(\mathrm{x}, \mathrm{y}, \mathrm{z})$.

$$
D=D_{\infty} e^{\beta \sum_{i=1}^{n} \frac{\xi_{i}}{\sqrt{\left(x-a_{i}\right)^{2}+\left(y-b_{i}\right)^{2}+\left(z-c_{i}\right)^{2}}}}
$$

Eq. (7) directly indicates that the diffusion coefficients can be smaller (i. e. under-diffusion) or greater (i. e. super-diffusion) than the free Brownian motion value $\mathrm{D}_{\infty}$ depending on the exponential part of the equation. Therefore, the conformational potential theory developed in this paper is consistent to the intracellular 'anomalous diffusion' observed in single particle tracking experiments.

\section{'Docking' and 'ejection' of molecules and the} networks of biological processes

It is aware that not all intracellular transport is achieved by random walk. As widely known, there are motor protein molecules responsible for intracellular transport of molecules from one location to another too ${ }^{29-31}$. Nevertheless, diffusion is inevitable even for such active transport as the cargo molecules have to bind to those transport proteins first which is achieved by diffusion or 'random walk'.

However, it is difficult to believe that systems as sophisticated as living organisms simply rely on 'random walk' to complete all the molecular binding in biological processes. How can orders be coded out of the 'randomness' or stochasticity? Conformational potential could probably offer an answer. If the 'random walk' is taken place in a potential gradient originated from the centre of the binding sites, a molecule will definitely find the binding sites, which can be seen as a 'stochastic gradient descent' process. 
Moreover, many macrobiomolecules contains not just one binding site. In many occasions, binding of a second molecule changes the conformation of the biomolecule therefore the first molecule falls off. Such a binding, conformation change and unbinding process underlines many biological processes like gene transcription and translation, cell metabolism and endocrinology. It can be realized through the regulation of conformational potentials, namely, prior to the binding of a second molecule, the first molecule are attracted to the biomolecule, upon binding of the second molecule, the conformation of binding site of the first molecule will be changed leading to its 'ejection' from the biomolecules (i. e. repulsion).

Like very sophisticated computers can be build up on the basis of 0 and 1. An overarching systematic network of biological processes could also be built from such a 'binding' and 'ejection' mechanism via regulation of conformational potentials on the molecular level. Similar to setting up chain reactions of dominos, the machinery of lives can be run by creating chain reactions of 'binding' and 'ejection' with regulated conformational potentials.

The conformational coefficient, conformational potential and relation to other fundamental theories

Firstly, although we defined and derived the conformational coefficient $\xi$, there might be more fundamental level constant contained in this coefficient as in the cases of gravitational potential and electrostatic potential where we have the gravitational constant and Coulomb's constant.

Secondly, although we developed the theory on the conformational potential based on purely classical view of mechanics, however, the actual foundation of the theoretical development here is thermal dynamics, given the deep and far reaching of thermal dynamics, whether there is a link between the conformational potential and relativity and quantum mechanics is not clear at this stage. The least known is that conformational coefficient has a unit of $\mathrm{kg} . \mathrm{m}^{3} / \mathrm{s}^{2}$ suggesting the conformation may be related to space and time or arrangement of space and time. As widely recognized, biomolecules such as proteins often have extraordinary properties that are difficult to be replicated with common artificial materials. Whether we are looking at a new type of fundamental force is also yet to be discovered.

\section{Conclusion}

In this paper, we developed a theory for the conformational interactions (i. e. binding and ejection) between molecules (e. g. common molecules or biomolecules) and biomolecules (e. g. DNA, RNA and proteins). This theory attributes the interactions to a postulation on existence of conformational potentials between conformationally matching molecules (attractive) and nonmatching molecules (repulsive).

Based on the postulation of conformational potentials, we derived an equation for spatially varied diffusion coefficients and the corresponding stochastic equation for diffusive motion of molecules in a conformational potential, which indicates the Einstein's diffusion theory (i. e. free Brownian diffusion) with invariant diffusion coefficient, popularly adopted for studying intracellular diffusion of biomolecules, is just an approximation of the diffusion theory derived here at places far from the centers of the binding sites. Our theory is consistent to the 'anomalous diffusion' observed in single particle tracking studies in which significant disagreement between experiments and Einstein's diffusion theory is observed.

The formulation of the intracellular diffusion coefficients here provides a path for experimental measurement of the conformational coefficient $\xi$ providing for the 
first time an approach to quantify 'conformation' of biomolecules and a footing for further development in physical or mathematical understanding of the mysterious conformation behaviors as well as the functioning mechanisms of biomolecules.

\section{References}

1. Dill, K. A.; MacCallum, J. L., The Protein-Folding Problem, 50 Years On. Science 2012, 338, 1042-1046.

2. Weiss, S., Measuring conformational dynamics of biomolecules by single molecule fluorescence spectroscopy. Nature Structural Biology 2000, 7 (9), 724-729.

3. Hammes, G. G.; Chang, Y.-C.; Oas, T. G., Conformational selection or induced fit: $A$ flux description of reaction mechanism. Proceedings of the National Academy of Sciences 2009, 106 (33), 13737.

4. Csermely, P.; Palotai, R.; Nussinov, R., Induced fit, conformational selection and independent dynamic segments: an extended view of binding events. Trends in Biochemical Sciences 2010, 35 (10), 539-546.

5. Rini, J. M.; Schulze-Gahmen, U.; Wilson, I. A., Structural evidence for induced fit as a mechanism for antibody-antigen recognition. Science 1992, 255 (5047), 959.

6. Sherman, W.; Day, T.; Jacobson, M. P.; Friesner, R. A.; Farid, R., Novel Procedure for Modeling Ligand/Receptor Induced Fit Effects. Journal of Medicinal Chemistry 2006, 49 (2), 534-553.

7. Ellis, R. J.; Minton, A. P., Join the crowd. Nature 2003, 425 (6953), 27-28.

8. Zimmerman, S. B.; Minton, A. P., Macromolecular Crowding: Biochemical, Biophysical, and Physiological Consequences. Annual Review of Biophysics and Biomolecular Structure 1993, 22 (1), 27-65.

9. Ellis, R. J., Macromolecular crowding: obvious but underappreciated. Trends in Biochemical Sciences 2001, 26 (10), 597-604.

10. Einstein, A., Über die von der molekularkinetischen Theorie der Wärme geforderte Bewegung von in ruhenden Flüssigkeiten suspendierten Teilchen. Annalen der Physik 1905, 322 (8), 549-560.

11. Kubo, R., The fluctuation-dissipation theorem.
Reports on Progress in Physics 1966, 29 (1), 255-284.

12. Schulten, K. Non-Equilibrium Statistical Mechanics. https://www.ks.uiuc.edu/Services/Class/PHYS498/.

13. von Smoluchowski, M., Zur kinetischen Theorie der Brownschen Molekularbewegung und der Suspensionen. Annalen der Physik 1906, 326 (14), 756-780.

14. Islam, M. A., Einstein-Smoluchowski Diffusion Equation: A Discussion. Physica Scripta 2004, 70 (2-3), 120-125.

15. Qian, H.; Sheetz, M. P.; Elson, E. L., Single particle tracking. Analysis of diffusion and flow in two-dimensional systems. Biophysical Journal 1991, 60 (4), 910-921.

16. Jaqaman, K.; Loerke, D.; Mettlen, M.; Kuwata, H.; Grinstein, S.; Schmid, S. L.; Danuser, G., Robust single-particle tracking in live-cell time-lapse sequences. Nature Methods 2008, 5, 695.

17. Kapanidis, A. N.; Uphoff, S.; Stracy, M., Understanding Protein Mobility in Bacteria by Tracking Single Molecules. Journal of Molecular Biology 2018, 430 (22), 4443-4455.

18. Shen, H.; Tauzin, L. J.; Baiyasi, R.; Wang, W.; Moringo, N.; Shuang, B.; Landes, C. F., Single Particle Tracking: From Theory to Biophysical Applications. Chemical Reviews 2017, 117 (11), 7331-7376.

19. Liu, Z.; Lavis, Luke D.; Betzig, E., Imaging Live-Cell Dynamics and Structure at the Single-Molecule Level. Molecular Cell 2015, 58 (4), 644-659.

20. Metzler, R.; Jeon, J.-H.; Cherstvy, A. G.; Barkai, E., Anomalous diffusion models and their properties: non-stationarity, non-ergodicity, and ageing at the centenary of single particle tracking. Physical Chemistry Chemical Physics 2014, 16 (44), 24128-24164.

21. Shav-Tal, Y.; Darzacq, X.; Shenoy, S. M.; Fusco, D.; Janicki, S. M.; Spector, D. L.; Singer, R. H., Dynamics of Single mRNPs in Nuclei of Living Cells. Science 2004, 304 (5678), 1797.

22. Spillane, K. M.; Ortega-Arroyo, J.; de Wit, G.; Eggeling, C.; Ewers, H.; Wallace, M. I.; Kukura, P., High-Speed Single-Particle Tracking of GM1 in Model Membranes Reveals Anomalous Diffusion due to Interleaflet Coupling and Molecular Pinning. Nano Letters 2014, 14 (9), 5390-5397.

23. Tabei, S. M. A.; Burov, S.; Kim, H. Y.; Kuznetsov, A.; 
Huynh, T.; Jureller, J.; Philipson, L. H.; Dinner, A. R.; Scherer, N. F., Intracellular transport of insulin granules is a subordinated random walk. Proceedings of the National Academy of Sciences 2013, 110 (13), 4911.

24. Kang, M.; DiBenedetto, E.; Kenworthy, Anne K., Proposed Correction to Feder's Anomalous Diffusion FRAP Equations. Biophysical Journal 2011, 100 (3), 791-792.

25. Smith, P. R.; Morrison, I. E. G.; Wilson, K. M.; Fernández, N.; Cherry, R. J., Anomalous Diffusion of Major Histocompatibility Complex Class I Molecules on HeLa Cells Determined by Single Particle Tracking. Biophysical Journal 1999, 76 (6), 3331-3344.

26. Dietrich, C.; Yang, B.; Fujiwara, T.; Kusumi, A.; Jacobson, K., Relationship of Lipid Rafts to Transient Confinement Zones Detected by Single Particle Tracking. Biophysical Journal 2002, 82 (1), 274-284.

27. Saxton, Michael J., Wanted: A Positive Control for Anomalous Subdiffusion. Biophysical Journal 2012, 103 (12), 2411-2422.

28. Saxton, M. J., Anomalous diffusion due to obstacles: a Monte Carlo study. Biophysical Journal 1994, 66 (2, Part 1), 394-401.

29. Hirokawa, N.; Noda, Y.; Tanaka, Y.; Niwa, S., Kinesin superfamily motor proteins and intracellular transport. Nature Reviews Molecular Cell Biology 2009, 10, 682.

30. Hirokawa, N., Kinesin and Dynein Superfamily Proteins and the Mechanism of Organelle Transport. Science 1998, 279 (5350), 519.

31. Dixit, R.; Ross, J. L.; Goldman, Y. E.; Holzbaur, E. L. F., Differential Regulation of Dynein and Kinesin Motor Proteins by Tau. Science 2008, 319 (5866), 1086. 